\title{
La production de chitine par les crustacés dans les écosystèmes marins
}

\author{
Charles Jeuniaux, Marie-Françoise Voss-Foucart et Jean-Claude Bussers \\ Laboratoire de morphologie, systématique et écologie animales, Institut \\ Van Beneden, quai Van Beneden, 22, B-4020 Liège, Belgique.
}

Reçu le 9 décembre 1992, accepté le 9 avril 1993.

Chitin production by crustaceans in marine ecosystems.

Jeuniaux Ch., M.-F. Voss-Foucart, J.-C. Bussers. Aquat. Living Resour., 1993, 6, 331-341.

Abstract

Chitin is synthesized by numerous animal species, either unicellular organisms or metazoans, belonging mainly to zoological groups of the Coelomate Spiralia lineage. However, the produced chitin in marine ecosystems is principally by crustaceans. A comparative study of analytical data so far available allowed calculation of chitin biomass and chitin production values in some types of marine ecosystems, and thus estimation of the quantitative importance of chitin in the biogeochemical cycles of carbon and nitrogen. The main data so far available concerns mediterranean plankton in Calvi bay (Corsica), arctic and antarctic krill, lobster population on South African coasts, and infralittoral benthic communities growing on rocky substrates on Corsica coasts. In all these cases, chitin production was estimated roughly at $1 \mathrm{~g}$ per year and per square meter of rocky substrate or sea surface. Taking into account these production values and the relative extent of the main marine ecosystems over the world, the total production of chitin due to marine crustaceans was estimated at about 2.3 billion metric tons per year.

Keywords: Chitin, Crustacea, marine environment.

Résumé

Bien que la chitine soit synthétisée par un grand nombre d'espèces animales, unicellulaires ou métazoaires, appartenant notamment aux groupes zoologiques de la lignée des Coelomates Spiralia, la production de chitine en milieu marin est essentiellement due aux Crustacés. Une étude comparée des quelques données analytiques disponibles a permis de tenter de calculer les valeurs de biomasse et de production de chitine dans quelques grands types d'écosystèmes marins, et d'évaluer ainsi l'importance quantitative de la chitine dans les cycles biogéochimiques du carbone et de l'azote. Les principales données actuellement disponibles concernent le plancton de la baie de Calvi (Corse) en Méditerranée, le krill arctique et antarctique, une population de langoustes des côtes de l'Afrique du Sud, et les communautés benthiques infralittorales à algues photophiles des côtes rocheuses de la Corse. Dans tous les cas, la production de chitine a pu être estimée à environ $1 \mathrm{~g}$ par an et par $\mathrm{m}^{2}$ de substrat rocheux ou de surface océanique. Sur base de ces valeurs de production et de l'étendue relative des principaux écosystèmes marins, la production totale annuelle de chitine par les crustacés marins a été évaluée à \pm 2.3 milliards de tonnes.

Mots-clés : Chitine, Crustacés, environnement marin.

\section{INTRODUCTION}

Il est important, pour l'écologiste, de considérer avec une attention particulière la place qu'occupe la chitine dans la biosphère et dans les cycles biogéochimiques, par rapport aux autres types de molécules organiques. En effet, la chitine

Aquat. Living Resour. 93/04 331 \$4.00/@ IFREMER-Gauthier-Villars est un matériel organique doué de propriétés physiques et chimiques peu banales, capable de résister à de nombreux facteurs physiques ou chimiques de dégradation de l'environnement naturel. C'est un homoglycane linéaire fait d'unités de $\mathrm{N}$-acétylglucosamine liées par des ponts $\beta .1,4$ glycosidiques. Comme la plupart des autres polysac- 
charides, la chitine offre une très haute résistance à l'hydrolyse enzymatique : celle-ci nécessite l'action d'hydrolases hautement spécifiques, en l'occurrence des chitinases ${ }^{1}$, plus précisément définies en tant que poly- $\beta-1,4$ (2-acétamido-2-deoxy)-D-glucoside glycanohydrolases (E. C.3.2.1.14). L'action de ces chitinases sur la chitine conduit à une hydrolyse partielle, les produits d'hydrolyse étant de petits oligomères de la N-acétylglucosamine, principalement du chitobiose et du chitotriose. Une hydrolyse complète en $\mathrm{N}$-acétylglucosamine libre requiert donc l'intervention d'un second type d'hydrolase : une chitobiase, ou de façon plus générale une $\beta$-N-acétylD-glucosaminidase (E. C.3.2.1.30).

La chitine apparait donc avec la cellulose comme un des matériaux organiques naturels les plus réfractaires, dont la place dans les cycles biogéochimiques de ses éléments constitutifs mérite d'autant plus d'être précisée que l'importance quantitative de ce polysaccharide dans les écosystèmes ne fait guère de doutes. La chitine est, en effet, largement distribuée chez les êtres vivants, non seulement dans le règne animal mais également dans tout le règne des Fungi (champignons, levures, moisissures) et dans bon nombre de protistes ou d'algues unicellulaires comme les Diatomées (Smucker, 1991). En fait, si on prend en compte la chitine des êtres vivants et celle des cadavres et des sédiments, on peut se demander si la chitine n'est pas, d'un point de vue quantitatif, le polysaccharide le plus important dans la biosphère, juste après la cellulose.

Mais, si on compare avec la cellulose, la production, la biodégradation et l'accumulation de la chitine dans les écosystèmes sont des étapes importantes des cycles biogéochimiques, non seulement du carbone, mais aussi de l'azote, puisque la proportion d'azote dans la molécule de chitine est d'environ $6 \%$.

L'idée suivant laquelle la chitine doit occuper une place importante dans les cycles biogéochimiques du carbone et de l'azote est admise volontiers par les écologistes, mais c'est surtout l'étude des processus de biodégradation qui a retenu l'attention des auteurs (Gooday, 1990). On sait depuis longtemps qu'il existe, dans les milieux marins et estuariens, de nombreuses espèces de micro-organismes capables de produire des chitinases et de dégrader la chitine (ex. : Zobell et Rittenberg, 1938; Seki et Taga, 1963; Seki, 1965, 1966 ; Okutani, 1975 ; Gooday, 1990). Les structures squelettiques chitineuses mortes (coquilles vides de mollusques, carapaces de crustacés après la mue ou la mort, etc.) sont rapidement colonisées puis perforées par de nombreuses espèces de micro-

(1) Seuls les lysozymes (E. C. :3.2.1.17) sont capables d'attaquer les liaisons $\beta-1,4$ au sein de la chitine, mais avec une vitesse de réaction enzymatique considérablement plus faible. organismes endolithiques, à savoir bactéries (notamment actinomycètes), cyanophycées, chlorophycées, ascomycètes, oomycètes, diatomées, qui dégradent les trames organiques chitinoprotéiques grâce à l'élaboration d'enzymes chitinolytiques (Poulicek, 1983; Poulicek et Jaspar-Versali, 1984 ; Poulicek et al., 1986). Par ailleurs, la chitine des formations squelettiques et cuticulaires des invertébrés marins est également rapidement hydrolysée et recyclée par les nombreuses espèces de prédateurs capables de sécréter des chitinases et des chitobiases au niveau du tube digestif (Jeuniaux, 1961, 1963; Micha et al., 1973; Jeuniaux et al., 1982). L'abondance et la diversité des organismes chitinolytiques en milieu marin, ainsi que l'efficacité et la rapidité relative de ces processus de biodégradation de la chitine permettent de comprendre pourquoi la chitine détritique ne s'accumule guère dans les sédiments marins, où les teneurs en chitine sont généralement très faibles, de l'ordre de $100 \mu \mathrm{g}$ par g de sédiment décalcifié (Poulicek et Jeuniaux, 1989; Gooday, 1990).

Si l'on connaît relativement bien aujourd'hui les modalités de la biodégradation de la chitine en milieu marin, il est par contre beaucoup plus difficile de fournir une évaluation précise des quantités de chitine produites et accumulées dans les écosystèmes et des organismes responsables de cette production. Le but du présent travail est de rassembler quelques-unes des données disponibles permettant de quantifier la production de chitine dans les écosystèmes marins, et de comparer la participation des Crustacés à celle des autres organismes producteurs de chitine dans les mêmes milieux. Nous tenterons enfin d'intégrer ces données dans une estimation de la production totale de chitine dans le biocycle marin.

Cette approche quantitative de ce qu'on pourrait appeler « l'écologie de la chitine » a été le thème d'un symposium dans le cadre du Congrès International

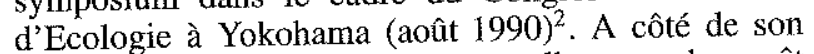
intérêt purement académique, une telle approche revêt aujourd'hui un intérêt pratique et technique d'autant plus grand que la chitine est de plus en plus utilisée dans l'industrie chimique et alimentaire, en médecine, en agronomie, etc.

En effet, la chitine (que les firmes industrielles isolent généralement à partir de carapaces de crustacés) sert de matière première pour la préparation du chitosane, un dérivé déacétylé qui possède des propriétés physiques et chimiques remarquables, mises à profit dans toute une série d'applications technologiques comme l'enrobage des graines et

(2) Cf. Jeuniaux et Voss-Foucart, 1991 ; Smucker, 1991 ; Miyamoto et al., 1991; Hirano et al., 1991 ; Poulicek et Jeuniaux, 1991 ; Gooday et al., 1991 ; Miller, 1991. 
des médicaments, la formation de gels pour la cosmétologie ou pour l'industrie alimentaire, la fixation des prothèses en chirurgie, la préparation de lentilles de contact et de membranes filtrantes, ou encore la fabrication de matériaux à haut pouvoir d'adsorption pour la chromatographie, pour la purification des eaux usées ou pour la récupération de protéines destinées à l'alimentation du bétail, etc. La conséquence de l'augmentation rapide de la demande de chitine purifiée et de chitosanes sur le marché est la recherche de nouvelles sources de chitine permettant un approvisionnement plus aisé ou plus régulier, ou des manutentions moins onéreuses. Mais il est bien connu que l'exploitation industrielle et commerciale d'une ressource naturelle (même renouvelable) doit être conduite sur des bases rationnelles, nécessitant notamment une étude préalable des biomasses disponibles et de la productivité, ainsi que de l'accessibilité de ces sources potentielles d'approvisionnement en chitine.

Une des conditions essentielles pour assurer la validité de ce genre d'étude est évidemment l'utilisation de méthodes de dosage quantitatif rigoureusement spécifiques, applicables non seulement aux crustacés mais aussi aux autres groupes d'organismes producteurs et aux peuplements biologiques dont ils font partie. Nous commencerons donc par décrire brièvement les méthodes de dosage de la chitine sur lesquelles reposent les données utilisées dans cette revue.

\section{MÉTHODES}

\section{Méthodes pour le dosage de la chitine}

La plupart des méthodes utilisées pour la mise en évidence de la chitine soit manquent de spécificité (comme la méthode dite «du chitosane » de Campbell, 1928), soit ne permettent pas un dosage quantitatif, comme la diffraction des rayons X (Rudall, 1963), l'analyse des spectres infrarouges (Darmon et Rudall, 1950 ; Pearson et al., 1960) ou les méthodes ultrastructurales basées sur la spécificité de certaines agglutinines par les polymères d'acétylglucosamine (Goldstein et al., 1975; Horisberger et Rosset, 1977).

Dans le cas des Crustacés, lorsqu'il s'agit de doser la quantité de chitine dans des organismes isolés ou a fortiori dans des structures isolées, la méthode dite « alkaline extraction » de Hackman $(1960,1982)$ permet d'obtenir des valeurs significatives, parce que la chitine est le seul constituant de la cuticule des Crustacés capable de résister aux traitements successifs par solutions acides et alcalines que comprend cette méthode. Cette méthode n'est pas appropriée dans le cas d'autres organismes, surtout s'ils ne sont pas isolés des autres composants du peuplement analysé.

Vol. $6, n^{\circ} 4-1993$
Une méthode plus spécifique est la méthode enzymatique, constituant à incuber le matériel étudié (après extraction préalable des sels calcaires et des protéines) en présence de chitinase purifiée jusqu'à hydrolyse complète, puis à doser les produits d'hydrolyse (Jeuniaux, 1963, 1965). Le matériel étudié est séché et pesé, puis décalcifié $(\mathrm{HCl} 0,5 \mathrm{~N})$, lavé et traité par $\mathrm{Na} \mathrm{OH} 0,5 \mathrm{~N}$ à $100^{\circ} \mathrm{C}$ pendant 3 à 6 heures pour libérer la chitine de ses complexes glycoprotéiques. Après lavage, le matériel résiduel est incubé à $\mathrm{pH} 5,2$ en présence de chitinase purifiée $(1 \mathrm{mg} / \mathrm{ml})$ pendant 3 heures ; l'opération est répétée jusqu'à hydrolyse complète. Les solutions surnageantes sont incubées à pH 5.2 et $37^{\circ} \mathrm{C}$ en présence de $\mathrm{N}$-acétyl-glucosaminidase (par addition de sérum de homard dilué) et l'acétylglucosamine libérée est mesurée par la méthode colorimétrique de Reissig et al. (1955).

Les chitinases utilisées pour le dosage enzymatique sont soit préparées et purifiées au laboratoire suivant la procédure de Jeuniaux (1958, 1959, 1966), soit achetées dans le commerce. Dans le premier cas, la chitinase est purifiée à partir de filtrats de cultures submergées de Streptomyces antibioticus sur milieu Czapeck sans glucose, à $\mathrm{pH} 7,5$ et $25^{\circ} \mathrm{C}$, contenant $10 \mathrm{~g}$ de chitine « colloïdale » (chitine de crevettes reprécipitée) par litre. Lorsque la teneur en chitinase est maximale, les suspensions sont filtrées sur laine de verre, et les filtrats sont acidifiés à $\mathrm{pH}$ 5.2. La chitinase est adsorbée sur chitine colloïdale à $0{ }^{\circ} \mathrm{C}$ et, après lavage, éluée par hydrolyse spontanée du substrat. La purification est achevée par deux précipitations fractionnées successives par le sulfate ammonique. La chitinase purifiée par ce procédé est exempte d'activité $\beta$ $\mathrm{N}$-acétylglucosaminidasique. D'autres procédés de purification ont été mis au point par divers auteurs, soit à partir de cultures de Streptomycètes (Berger et Reynolds, 1958 ; Ohtakara, 1971) soit à partir d'autres souches microbiennes, notamment Serratia marcescens (Roberts et Cabib, 1982). Diverses firmes commercialisent des préparations de chitinases purifiées par l'une ou l'autre de ces méthodes (Koch-Light, Serva, Sigma, USBC, Calbiochem). Ces chitinases, dont le pH optimum varie de 5.2 à 7.2 selon l'origine, sont des endochitinases dont les produits d'hydrolyse sont essentiellement des dimères et des trimères de l'acétylglucosamine.

Les résultats obtenus par la méthode enzymatique peuvent être exprimés en mg de chitine par unité de poids sec ou de poids décalcifié de matériel de départ, en appliquant un facteur de correction de 0.92 pour tenir compte de la perte d'une molécule d'eau par pont glycosidique au sein de la molécule de chitine. Si cette méthode présente les meilleures garanties de spécificité, elle donne cependant des résultats par défaut, légèrement inférieurs à la réalité, et ce pour deux raisons : d'une part, elle ne dose que l'acétylglucosamine libérée, et non la glucosamine, dont les chaînes de chitine peuvent 
contenir une certaine quantité, soit naturellement (Giles et al., 1958), soit à la suite des traitements subis antérieurement ; d'autre part, lorsque la chitine existe sous une forme hautement cristalline ou lorsqu'elle est liée à des scléroprotéines, il est parfois difficile d'obtenir une hydrolyse enzymatique complète.

\section{Calcul de la production de chitine par les communautés zooplanctoniques}

Les valeurs de la densité numérique et de la biomasse de chaque stade de chaque espèce de crustacé planctonique au cours d'un cycle annuel ont été établies par Dauby (1985) pour le plancton de la baie de Calvi (Corse) en Méditerranée. Ces valeurs sont exprimées par $\mathrm{m}^{2}$ de surface d'eau pour une colonne d'eau de 100 mètres de hauteur. Connaissant la teneur en chitine par rapport au poids sec total des différents stades des espèces concernées, la production journalière de chitine par espèce (ou groupe d'espèces) a été calculée par la méthode de Winberge (1971) (« cumulative growth method»), adaptée au cas d'espèces présentant une succession de stades larvaires différents durant leur vie, c'est-à-dire :

$$
\mathrm{P} \sum_{n=1}^{i} \frac{\mathrm{Nn} \cdot \Delta \overline{\mathrm{W}} \mathrm{n} \cdot C n}{t n}
$$

où $i$ est le nombre de stades larvaires successifs, Nn le nombre d'individus de chaque stade (par unité de surface), $\Delta \vec{W}_{n}$ l'accroissement de poids pendant le stade $n, C n$ la proportion de chitine en $\%$ du poids sec, et $t n$ la durée du stade $n$. Cette formule ne permet pas de prendre en compte la production d'exuvies, de sorte que les valeurs de production de chitine calculées sont des valeurs par défaut. Il faut noter cependant que, chez les crustacés planctoniques, l'exuvie ne représente qu'une faible partie de la cuticule de l'animal, une part importante de celleci étant hydrolysée et récupérée au cours de la période de mue qui précède l'exuviation.

\section{DISTRIBUTION DE L'APTITUDE A LA BIOSYNTHESE DE CHITINE CHEZ LES ORGANISMES MARINS}

Bien que l'organisation supramoléculaire de la chitine puisse se présenter sous des formes cristallographiques différentes: $\alpha, \beta, \gamma$, il semble bien que sa structure moléculaire soit identique dans tous les cas où elle a été étudiée, et que sa biosynthèse soit contrôlée par les mêmes systèmes enzymatiques, à savoir des chitine-synthases (E. C. 2.4.1.16) qui catalysent le transfert d'une unité d'acétylglucosamine à partir de 1'UDPAG (uridinediphosphate-N-acétylglucosamine) sur un récepteur du type chitodextrine. Il y a donc beaucoup de chance pour que les systèmes biosynthétiques de la chitine soient homologues à travers l'ensemble des règnes vivants.

La biosynthèse de la chitine est présente de manière à peu près générale chez les Fungi, y compris chez les moisissures et les levures. Ces organismes étant peu abondants dans les biocénoses marines, leur contribution à la production de chitine dans les milieux marins est donc, à première vue, d'importance mineure.

Si la biosynthèse de la chitine manque totalement chez les Végétaux Métaphytes comme chez les Procaryotes (bactéries et algues bleues), elle est présente chez un certain nombre d'espèces d'eucaryotes unicellulaires, comme les Rhizopodes (Sachs, 1954), les Foraminifères (Hedley, 1962), les Cnidosporidies (Vavra, 1967), chéz bon nombre d'espèces de Ciliés, au niveau des membranes kystiques (Bussers et Jeuniaux, 1974) ainsi que chez les Diatomées (Smucker, 1991). Les Diatomées, par leur importance quantitative dans le plancton marin comme dans les couvertures biologiques benthiques, contribuent donc de façon significative à une production « primaire » de chitine (Smuker, 1991).

L'utilisation de la chitine comme matrice organique de structures squelettiques ou cuticulaires est répandue de manière très inégale dans la série animale (Jeuniaux, 1971, 1982b). Présente déjà chez divers groupes de Protozoaires, dont les Ciliés déjà cités, la synthèse de la chitine fait certainement défaut chez les Spongiaires (Dauby et Jeuniaux, 1986) mais est largement mise à profit par les Cnidaires pour l'édification du périsarc chez les Hydrozoaires Calypto-et Gymnoblastiques, les Chondrophiidae, certains Octocoralliaires Stolonifères, et certains scyphistomes de Scyphozoaires, mais elle manque chez les autres groupes de Cnidaires et chez les Cténaires. Chez les Triblastiques, on ne connaît aucune formation de nature chitineuse chez les Plathelminthes ni chez les Némertiens. Par contre, la grande majorité des classes de Triblastiques de la lignée des Spiralia («Protostomiens») utilise la chitine soit pour l'édification de l'enveloppe cuticulaire (Arthropodes, Tardigrades et Onychophores, ainsi que les Kinorhynques), pour l'élaboration de la coquille (Mollusques) pour la fabrication des soies (Annélides, Echiuriens) ou pour la construction de tubes (Vestimentifères et Pogonophores), tandis que chez les Aschelminthes (Rotifères, Gastrotriches, Nématodes), la chitine intervient dans la construction d'une ou plusieurs enveloppes de l'oeuf. Les Lophophoriens, dont la position phylogénétique reste des plus controversée, font également usage de la chitine comme matériau de construction, soit au niveau des ectocystes des Bryozoaires Ectoproctes, soit au niveau des tubes des Phoronidiens, soit encore dans la coquille des Brachiopodes Inarticulés et dans la cuticule du pédoncule chez les Brachiopodes Articulés.

$\mathrm{Au}$ contraire, dans la lignée des Archicoelomates («Deutérostomiens »), qui comprend les Echino- 
dermes, les Stomochordés, les Céphalochordés, les Tuniciers et les Chordés, la synthèse de chitine semble avoir été totalement perdue, à l'exception des Tuniciers qui l'utilisent pour la formation de leur membrane péritrophique (chez Phallusia, Ciona, Halocynthia, et Clavellina: Peters, 1966, 1967).

On voit donc qu'une vingtaine de grandes classes d'animaux marins, y compris les Crustacés, sont susceptibles de contribuer à la production et l'accumulation de chitine dans les écosystèmes marins. L'importance de la contribution d'un groupe ou d'une espèce dépend évidemment de l'importance quantitative de ses populations dans l'écosystème considéré et de la vitesse de production par individu et par stade de développement. De plus, il faut tenir compte de la variabilité, parfois considérable, de la teneur en chitine d'un même type de structure d'une espèce à l'autre au sein d'un même groupe (Hydrozoaires : Jeuniaux, 1963 ; Mollusques : Goffinet et Jeuniaux, 1979 ; Bryozoaires : Jeuniaux, 1982a). Dans ces conditions, il serait illusoire d'essayer d'obtenir une estimation chiffrée de la biomasse de chitine dans un écosystème donné sans passer par une mesure directe, in situ. Il en va de même pour la mesure de la production.

Des données analytiques des biomasses de chitine et des estimations de sa production ont été obtenues dans un nombre limité de communautés marines: le zooplancton méditerranéen et les communautés benthiques croissant sur substrats durs sur le littoral de Corse. D'autres valeurs ont pu être calculées, par extrapolation, à partir de l'étude de populations de krill et de langouste.

\section{BIOMASSES ET PRODUCTION DE CHITINE DANS UNE COMMUNAUTÉ ZOOPLANC- TONIQUE DE MÉDITERRANÉE}

Une étude quantitative du cycle annuel complet du zooplancton de Méditerranée a été réalisée par Dauby (1985), à partir de la station océanographique «Stareso », dans la baie de Calvi (Corse). Ce plancton est dominé à $90 \%$ par les Crustacés Copépodes et Cladocères pendant la plus grande partie de l'année. Les variations de biomasse et de production pour chaque espèce ont été établies.

Par ailleurs, nous avons mesuré la teneur en chitine de chaque espèce dominante par la méthode enzymatique (Gervasi et al., 1988). Nous avons constaté que pour une espèce donnée, la teneur en chitine reste relativement constante pendant le développement larvaire et ne diffère guère de celle du stade adulte. Par contre, d'importantes variations ont été constatées d'une espèce à l'autre : chez les Copépodes, les valeurs de chitine, exprimées en \% du poids sec total, varient de $3.1 \%$ chez Clausocalanus arcuicornis et $C$. furcatus à $8,58 \%$ chez Acartia clausi. Dans le cas des Cladocères, la teneur en chitine de l'espèce dominante, Evadne spinifera est de 12,22\%.
A partir de ces valeurs analytiques et des données de Dauby (1985) sur les variations de densité numérique et de biomasse de chaque stade de chaque espèce au cours du cycle annuel, la production moyenne annuelle de chitine calculée pour l'ensemble des Copépodes et des Cladocères de la baie de Calvi est de $\pm 1 \mathrm{~g}$ par $\mathrm{m}^{2}$ par an, pour une colonne d'eau de $100 \mathrm{~m}$ de profondeur, ce qui correspond à une biomasse moyenne annuelle de $\pm 26 \mathrm{mg}$ par $\mathrm{m}^{2}$ (Gervasi et al., 1988). Les variations saisonnières sont considérables : une production maximum de chitine, soit $20,06 \mathrm{mg}$ par $\mathrm{m}^{2}$ et par jour a été enregistrée au mois de mai, et correspondait à une biomasse de $414 \mathrm{mg}$ par $\mathrm{m}^{2}$.

\section{PRODUCTION DE CHITTINE PAR LES EU. PHIAUSIACES (KIRILL)}

Le krill antarctique, Euphausia superba, fait l'objet de pêcheries industrielles depuis 1960 pour la commercialisation de la chair de l'abdomen, et plusieurs auteurs recommandent l'utilisation des déchets de ces pêches au krill pour la production industrielle de chitine (Anderson et al., 1978 ; Brzeski, 1982). La production annuelle de krill a été estimée de 16 à 1350 millions de tonnes par an (en poids frais) par Everson (1977) et plus modestement de 100 à 500 millions de tonnes par an (toujours en poids frais) par Ross et Quétin (1988). Ces estimations sont cependant basées sur des indications indirectes, comme le rendement des efforts de pêche, et non sur une mesure directe des biomasses et de la production. On peut esayer de calculer de manière plus précise la production de chitine par les Euphausiasés en se basant sur les données de Lindley (1982) qui a mesuré les biomasses et productions globales des principales espèces de krill dans l'Atlantique Nord et différentes mers qui en dépendent. Il suffit en effet de transformer les données de Lindley en admettant que la teneur en chitine doit être voisine de celle mesurée par Yanase (1975) chez Euphausia superba, à savoir 38,7\% du poids sec de la cuticule, soit $7,08 \%$ du poids sec total.

Tenant compte de ces valeurs, nous avons calculé (Jeuniaux et Voss-Foucart, 1991) que les biomasses de chitine de krill varient en général de 0,21 à $0,37 \mathrm{mg}$ par $\mathrm{m}^{3}$ d'eau, la valeur maximum enregistrée étant de $0,62 \mathrm{mg}$ par $\mathrm{m}^{3}$ dans la mer de Norvège, pour une population de krill dominée par Meganyctiphanes norvegicus. La production de chitine calculée varierait de 0,24 à $0,28 \mathrm{mg} \mathrm{par} \mathrm{m}^{3}$ et par an, dans la plupart des régions, mais dépasserait $0,85 \mathrm{mg}$ par $\mathrm{m}^{3}$ par an en mer de Norvège, mer du Nord, Nova Scotia et Iberia Shelfs.

Toutes ces valeurs sont exprimées par mètres cubes d'eau pompée à 10 mètres de profondeur. $\mathrm{Si}$ on extrapole ces valeurs à une colonne d'eau de $100 \mathrm{~m}$ d'épaisseur, tout en tenant compte, comme hypothèse de travail, d'une distribution discontinue telle que la colonne d'eau n'est occupée qu'à moitié, les biomasses 
de chitine du krill varieraient de 10,5 à $31 \mathrm{mg}$ par $\mathrm{m}^{2}$ et la production de chitine par les Euphausiacés pourrait être de 12 à $42 \mathrm{mg}$ par $\mathrm{m}^{2}$ par an.

On remarquera que ces valeurs de biomasse de chitine de krill sont du même ordre de grandeur que celles que nous avons obtenues pour les Copépodes et les Cladocères du plancton méditerranéen, mais que les valeurs de production sont nettement inférieures. Elles ne tiennent pas compte, par ailleurs, de la production de chitine au niveau des exuvies.

Pour estimer les quantités de chitine qui peuvent être produites par le krill, nous disposons également des données publiées par Ritz et Hozie (1982) sur une communauté d'Euphausiacés du Pacifique Sud dominée par l'espèce Nyctiphanes australis, où la production d'exuvies est prise en compte. Nous avons calculé (Jeuniaux et Voss-Foucart, 1991), toujours sur base d'une teneur en chitine de $7,08 \%$ du poids sec total obtenue par Yanase pour E. superba, que la production de chitine, calculée sur base des biomasses du stade adulte, serait de $5.6 \mathrm{mg}$ par $\mathrm{m}^{3}$ et par an, soit $283 \mathrm{mg}$ par $\mathrm{m}^{2}$ et par an (pour une colonne d'eau de $100 \mathrm{~m}$ d'épaisseur) et la production due aux exuvies serait de $15,87 \mathrm{mg}$ par $\mathrm{m}^{3}$ et par an, soit $794 \mathrm{mg}$ par $\mathrm{m}^{2}$ et par an. La production totale annuelle (adultes + exuvies) de chitine par le krill du Pacifique Sud pourrait donc être estimée à $1,077 \mathrm{~g}$ par $\mathrm{m}^{2}$ et par an, une valeur très proche de celle obtenue pour la production de chitine par les Crustacés du zooplancton méditerranéen.

\section{PARTICIPATION DES CRUSTACÉS A LA CONSTITUTION DES BIOMASSES DE CHI- TINE DANS LES COMMUNAUTES BEN- THQQES DES SUBSTRATS ROCHEUX DU LITTORAL MÉDITERRANÉEN}

Contrairement aux écosystèmes planctonique et pélagique, dont la faune est tellement riche en Crustacés qu'on peut affirmer que l'énorme majorité de la chitine produite résulte de l'activité biosynthétique de ceux-ci, les communautés benthiques sur substrat rocheux des zones infralittorales sont constituées par une flore et une faune beaucoup plus diversifiées. Dans de tels cas, la part prise par les différents groupes zoologiques à la production de chitine et à la constitution de la biomasse de ce polysaccharide est impossible à préciser de prime abord. Nous avons cherché à répondre à cette question par une analyse détaillée de la biomasse de la couverture biologique d'un tombant vertical, à 22 mètres de profondeur, sur la côte nord de la Corse, entre Calvi et la pointe de la Revellata (au lieu-dit Bibliothèque). Le peuplement analysé correspond à une biocénose à algues photophiles, faciès à Cystoseira stricta et Cystoseira balearica (V. Demoulin, comm. pers.).
Une surface carrée de $0,5 \mathrm{~m}$ de côté a été entièrement scalpée par des plongeurs en scaphandre autonome, et la couverture biologique a été soigneusement récoltée à l'aide d'une suçeuse à air comprimé (Bussers et al., 1983) qui permet de récolter aussi la faune vagile vivant parmi les algues. Le matériel a été trié, réparti par groupes zoologiques, lavé, séché, pesé, décalcifié et utilisé pour le dosage de la chitine par méthode enzymatique (tabl. 1). On voit que, si on fait abstraction de l'abondant matériel biologique encroûtant qui n'a pas pu être détaché des frondes des algues, (et qui comprend notamment beaucoup de Cirripèdes), ce sont les Crustacés qui interviennent de manière prépondérante dans la biomasse de chitine de la faune isolée $(65 \%)$. Les Spongiaires, qui représentent plus de la moitié du poids sec des organismes isolés, contribuent apparemment à la biomasse de chitine de la faune isolée à concurrence de $20 \%$. Mais une étude plus poussée de l'origine de la chitine dosée dans ces éponges a montré (Dauby et Jeuniaux, 1986) que celle-ci est exclusivement d'origine exogène elle provient des organismes épibiontes et surtout endobiontes qui fourmillent dans les cavités de ces éponges. Or, ces organismes sont, en grande majorité, des Crustacés de petite taille (Ostracodes, Copépodes Harpacticoïdes, Amphipodes, Isopodes, Mysidacés, Cirripèdes, etc.). Les autres organismes ne participent à la biomasse de chitine de la faune isolée que de façon modeste, ou font partie de la couverture biologique encroûtante qui n'a pas été détachée des frondes (c'est le cas surtout des Bryozoaires et des Hydrozoaires).

La prédominance des Crustacés dans la constitution de la biomasse de chitine de ce type de peuplement est également mise en évidence dans les résultats d'une série d'analyses, présentés dans le tableau 2, réalisées également sur les côtes du Nord de la Corse. Divers échantillons de communautés infralittorales appartenant à la même biocénose d'algues photophiles dominées par Cystoseira stricta et $C$. balearica ont été grattés et récoltés en plongée à des profondeurs différentes sur le tombant rocheux (de 5 à $30 \mathrm{~m}$ de profondeur). Le matériel biologique récolté a été trié et séparé en deux portions, l'une constituée par les Crustacés benthiques ou vagiles de taille supérieure à $10 \mathrm{~mm}$ (surtout des Décapodes), l'autre contenant le reste du matériel (y compris les Crustacés de trop petite taille ou les espèces encroûtantes : Balanes...). Les déterminations de poids $\mathrm{sec}$ et de chitine ont été conduites séparément sur les 2 portions de matériel.

La biomasse de chitine, non compris les Crustacés Décapodes, est relativement constante et varie, quelle que soit la profondeur, de 400 à $900 \mathrm{mg}$ par m² (tabl. 2). La présence de Crustacés Décapodes de taille supérieure à $10 \mathrm{~mm}$ est aléatoire ; toutefois, à l'exception d'un échantillon prélevé à 30 mètres, leur contribution à la biomasse de chitine est importante et souvent du même ordre de grandeur que la contribution de l'ensemble des autres organismes. 
Tableau 1. - Biomasses de chitine constituées par les principaux groupes zoologiques d'une biocénose à algues photophiles, faciès à Cystoseira stricta et $C$. balearica (Calvi, «Bibliothèque », tombant vertical, $-22 \mathrm{~m}$ ).

\begin{tabular}{|c|c|c|c|c|}
\hline & $\begin{array}{l}\text { Poids calcifié } \\
\left(\mathrm{mg} / 0,25 \mathrm{~m}^{2}\right)\end{array}$ & $\begin{array}{l}\text { Poids décalcifié } \\
\left(\mathrm{mg} / 0,25 \mathrm{~m}^{2}\right)\end{array}$ & $\begin{array}{l}\text { Chitine (totale) } \\
\left(\mathrm{mg} / 0,25 \mathrm{~m}^{2}\right)\end{array}$ & $\begin{array}{c}\text { Chitine } \\
\text { (mg/g pds décalcifié) }\end{array}$ \\
\hline \multicolumn{5}{|l|}{ Faune isolée } \\
\hline Crustacés (sauf Paguridés) & 76 & 27 & 5,4 & 195 \\
\hline Bryozoaires & 714 & 26 & 0,6 & 22 \\
\hline Spongiaires & 4938 & 3242 & 1,73 & 0,46 \\
\hline Gastéropodes & 851 & 39 & 0,18 & 4,7 \\
\hline Lamellibranches & 419 & - & 0,07 & - \\
\hline Annélides (et tubes) & 231 & 15 & 0,08 & 5,5 \\
\hline Paguridés (+ coquilles) & 274 & - & 0,04 & - \\
\hline Echinodermes & 68 & 11 & 0 & 0 \\
\hline Foraminifères & 159 & 9 & 0,03 & 3,2 \\
\hline Divers & 98 & 24 & 0,16 & 6,7 \\
\hline Total faune isolée & 7828 & $3393\left({ }^{1}\right)$ & 8,29 & - \\
\hline \multicolumn{5}{|l|}{ Cystoseira spp. } \\
\hline Frondes & 68070 & 23368 & 49,45 & 2,11 \\
\hline Stipes & 9036 & 4881 & 0,14 & 0,03 \\
\hline Sable & 12149 & 3509 & 3,29 & 0,94 \\
\hline
\end{tabular}

$\left.{ }^{1}{ }^{1}\right)$ le poids décalcifié n’a pas été mesuré pour les Lamellibranches et les Paguridés.

Tableau 2. - Communautés infralittorales à algues photophiles des côtes rocheuses du nord-ouest de la Corse : biomasses totales, biomasses de chitine et contribution relative des crustacés décapodes aux biomasses de chitine.

\begin{tabular}{|c|c|c|c|c|c|}
\hline \multirow{2}{*}{ Profondeur } & \multirow{2}{*}{$\begin{array}{c}\text { Nombres } \\
\text { d'échantillons }\end{array}$} & \multirow{2}{*}{$\begin{array}{c}\text { Biomasse } \\
\text { totale } \mathrm{g} / \mathrm{m}^{2}\end{array}$} & \multicolumn{3}{|c|}{ Biomasse de chitine en $\mathrm{mg} / \mathrm{m}^{2}$} \\
\hline & & & $\begin{array}{l}\text { Crustacés } \\
\text { exclus }\end{array}$ & $\begin{array}{l}\text { Crustacés } \\
\text { décapodes }\end{array}$ & Total \\
\hline $5-7 \mathrm{~m}$ & 3 & $2011 \pm 525$ & $615 \pm 245$ & $684 \pm 975$ & $1299 \pm 1208$ \\
\hline $9-11 \mathrm{~m}$ & 4 & $2336 \pm 1091$ & $915 \pm 260$ & $455 \pm 403$ & $1393 \pm 522$ \\
\hline $13 \mathrm{~m}$ & 1 & 1261 & 395 & 135 & 530 \\
\hline $17 \mathrm{~m}$ & 1 & 1877 & 864 & 906 & 1760 \\
\hline $19-20 \mathrm{~m}$ & 4 & $2039 \pm 1024$ & $633 \pm 345$ & $120 \pm 145$ & $753 \pm 263$ \\
\hline $30 \mathrm{~m}$ & 1 & 2726 & 750 & 0 & 750 \\
\hline
\end{tabular}

Les analyses réalisées sur la côte nord ouest de la Corse ont permis de conclure que les biomasses moyennes de chitine des communautés infralittorales à algues photophiles des faciès à Cystoseira stricta et C. balearica sont de l'ordre de 1,10 g par $\mathrm{m}^{2}$ (Jeuniaux et al., 1982), et que les Crustacés y contribuent pour plus de 50\% (sans tenir compte des grandes espèces de Décapodes vivant dans les crevasses et anfractuosités). Des valeurs voisines $\left( \pm 1,4 \mathrm{~g}\right.$ par $\left.\mathrm{m}^{2}\right)$, mais beaucoup plus variables ont été mesurées pour les communautés benthiques sciaphiles des grottes semi-obscures, mais la part prise par les Crustacés y est beaucoup plus variable (Jeuniaux et al., 1982).

\section{PRODUCTION DE CHITINE PAR LES COM- MUNAUTÉS BENTHIQUES}

Nous n'avons pu aborder la mesure de la production de chitine par les communautés marines benthiques in situ que par le biais d'un artifice expérimental,

Vol. 6, $\mathrm{n}^{\circ} 4-1993$ consistant à mesurer la quantité de chitine accumulée, après un laps de temps donné, par la couverture biologique colonisant des substrats nus, immergés en mer à des profondeurs diverses.

Cette étude (Jeuniaux et al., 1988, 1989) a été conduite pendant plusieurs années d'affilée sur les côtes de la pointe de la Revellata, près de la Station Stareso à Calvi (Corse). Les substrats consistaient en plaques rectangulaires, faites de granite, de terre cuite, de verre ou de divers types de plastiques. Plusieurs lots de plaques (168 au total) ont servi à cette série d'analyses. Le matériel biologique de chaque plaque, récolté au terme d'une période donnée, est lavé, pesé, broyé et utilisé pour le dosage de la chitine par méthode enzymatique. Les résultats sont exprimés par unité de surface $\left(\mathrm{m}^{2}\right)$ et par unité de temps, et sont considérés comme correspondant à la mesure de la «production nette » de chitine par ce type de peuplement dont la composition spécifique n'est pas fondamentalement différente de celle des communautés infralittorales 
Tableau 3. - Bilan de la production de chitine par les crustacés dans les écosystèmes marins.

\begin{tabular}{|c|c|c|c|c|}
\hline \multirow{2}{*}{$\begin{array}{l}\text { Écosystème } \\
\text { et communautés }\end{array}$} & \multirow{2}{*}{$\begin{array}{c}\text { Surface } \\
\text { occupée }\left({ }^{1}\right) \\
\times 10^{6} \mathrm{~km}^{2}\end{array}$} & \multicolumn{2}{|c|}{ Production annuelle de chitine $\left(\mathrm{g} / \mathrm{m}^{2}\right)$} & \multirow{2}{*}{$\begin{array}{c}\text { Totale } \\
\text { Crustacés } \\
\text { tonnes. } 10^{3} / \mathrm{an} \\
\end{array}$} \\
\hline & & Globale & Crustacés & \\
\hline Pélagique & 360 & & & \\
\hline Zooplancton de surface $\left({ }^{2}\right)$ & & 1,0 & 1,0 & 360000 \\
\hline Krill $\left({ }^{3}\right)$ & & 5,3 & 5,3 & 1938000 \\
\hline Crevettes & & $?$ & & \\
\hline \multicolumn{5}{|l|}{ Benthos } \\
\hline Rocheux littoral & 1,4 & & & \\
\hline Couverture biologique $\left({ }^{4}\right)$ & & 1,0 & 0,5 & 700 \\
\hline $\begin{array}{l}\text { Grands Crustacés } \\
\text { Décapodes }\left({ }^{5}\right)\end{array}$ & & 1,5 & 1,5 & 2100 \\
\hline Talus continental & 30,6 & $?$ & $?$ & $?$ \\
\hline Sédiments & 320 & & & \\
\hline Grands Décapodes $\left({ }^{5}\right)$ & & $?$ & $?$ & $?$ \\
\hline Total & & & & 2300800 \\
\hline \multicolumn{5}{|c|}{$\begin{array}{l}\text { (1) d'après Milliman (1974) et Tchernia (1979). } \\
\left({ }^{2}\right) \text { d'après les données de Gervasi et al. (1988), pour une colonne d'eau de } 100 \mathrm{~m} \text {. } \\
\left({ }^{3}\right) \text { calculées d'après les données de Ritz et Hozie (1982), ramenées au } \mathrm{m}^{2} \text { de surface et extrapolées pour une } \\
\text { colonne d'eau de } 500 \mathrm{~m} \text {. } \\
\left({ }^{4}\right) \text { d'après les données de Jeuniaux et al. (1988, 1989). } \\
\left({ }^{5}\right) \text { calculées en postulant la présence de populations monospécifiques de Crustacés Décapodes de grande taille } \\
\text { ayant une production équivalente à celle de Panulirus homarus (Berry et Smale, 1980). }\end{array}$} \\
\hline
\end{tabular}

à algues photophiles, voisines du site expérimental choisi.

La production de chitine, lente au cours de la première année d'immersion des plaques $(0,3 \mathrm{~g}$ par $\mathrm{m}^{2}$ par an à $\left.-18 \mathrm{~m}\right)$, devient plus importante $(1 \mathrm{~g}$ par $\mathrm{m}^{2}$ par an à $-18 \mathrm{~m}$ ) au cours de la deuxième année d'immersion, au moment où, apparemment, la communauté n'évolue plus guère. La production de chitine est plus faible $\left(0,34 \mathrm{~g}\right.$ par $\mathrm{m}^{2}$ par an) à une profondeur plus élevée $(-37 \mathrm{~m})$. Elle est plus faible sur verre que sur granite ou sur terre cuite. Des essais réalisés au moyen de divers types de plastiques ont montré que, sur ces substrats artificiels, la production de chitine par les communautés benthiques pionnières est de même importance que sur plaques de granite ou de terre cuite (Voss-Foucart et al., 1991), soit environ $1 \mathrm{~g} \mathrm{par} \mathrm{m}^{2}$ et par an.

Etant donné la part que prennent les Crustacés dans les biomasses de chitine de ce type de peuplement benthique (tableau 2), la production de chitine due aux seuls Crustacés peut être évaluée à la moitié de la production totale, soit $0.5 \mathrm{~g}$ par $\mathrm{m}^{2}$ par an. Cette estimation ne prend pas en compte la production due aux grandes espèces de Décapodes vivant dans les anfractuosités. Le cas d'une d'entre elles a été envisagé en détail par Berry et Smale (1980), qui ont étudié une population naturelle de langouste épineuse, Panulirus homarus, habitant les fonds rocheux d'un îlot isolé sur la côté du Natal en Afrique du Sud. Ces auteurs ont mesuré la production globale, la production de cuticule et celle des exuvies, et ont déterminé la teneur en chitine à différents stades, ce qui permet de calculer une production moyenne annuelle de chitine égale à $1.5 \mathrm{~g}$ par $\mathrm{m}^{2}$ et par an, valeur proche de celle que nous avons mesurée pour l'ensemble de la communauté benthique pionnière sur substrat rocheux en Méditerranée.

\section{ESSAI DE BILAN DE LA PRODUCTION TOTALE ANNUELLE DE CHITINE PAR DES CRUSTACÉS MARINS}

Sur base des données en notre possession, que nous venons de synthétiser, un premier essai de bilan de la production totale de chitine en milieu marin a été dressé (Jeuniaux et Voss-Foucart, 1991) ${ }^{3}$. Nous proposons ici un bilan quelque peu différent ( $t a b l .3)$, que nous avons calculé en reconsidérant certains postulats, et en mettant en évidence la contribution propre des Crustacés.

Pour établir ce bilan, nous avons postulé que la production de chitine par le plancton marin est partout du même ordre de grandeur que celle que nous avons mesurée en Méditerranée dans la baie de Calvi. Cette hypothèse n'est pas hasardeuse puisque le zooplancton de Méditerranée est réputé moins productif que celui d'autres mers ou océans, et que la dominance des Crustacés Copépodes, Ostracodes

(3) Une erreur s'est glissée dans le tableau 4 de cette publication (lire, en tête de la dernière colonne : «Tons $x 10^{3} \cdot \mathrm{yr}^{-1}$ » au lieu de «Tons $\mathrm{yr}^{-1}$ ». 
et des larves de Décapodes se vérifie pratiquement partout, sauf à certaines périodes bien particulières. Nous avons fait la même hypothèse en ce qui concerne l'ordre de grandeur de la production de chitine par les communautés benthiques recouvrant des substrats rocheux, en admettant (ce qui n'est certainement pas correct) que les valeurs mesurées à $-18 \mathrm{~m}$ de profondeur sont généralisables entre 0 et $200 \mathrm{~m}$.

La part des Crustacés à la production de chitine par la couverture biologique a été évaluée à $50 \%$. Il faut y ajouter la production due aux grandes espèces de Crustacés Décapodes vivant dans les crevasses et anfractuosités. Nous avons postulé la présence sur toutes les côtes rocheuses, entre 0 et $200 \mathrm{~m}$, d'au moins une population d'une espèce de grande taille de Crustacé Décapode dont la production de chitine serait comparable à celle de Panulirus homarus, mesurée par Berry et Smale (1980).

Pour le krill, nous avons extrapolé à une colonne d'eau de $500 \mathrm{~m}$, les valeurs calculées à partir des données de Ritz et Hozie (1982), les essaims d'Euphausiacés étant connus pour leurs migrations sur toute cette hauteur de colonne d'eau. Toutefois, compte tenu de la distribution hétérogène de ces essaims, nous avons corrigé la valeur calculée en divisant par un facteur 2. Les données de Ritz et Hosie ont été retenues de préférence à celles de Lindley (1982), parce que celles-ci ne prennent pas en compte la production d'exuvies.

Le tableau 3 met bien en évidence l'absence de données analytiques fiables sur la production de chitine par plusieurs communautés de Crustacés dont la production de chitine est certainement importante : notamment les bancs de crevettes et les associations de grands Crabes des fonds meubles de l'Arctique et du Pacifique Nord, sièges de pêcheries prospères. Notre évaluation de la production totale de chitine par les Crustacés marins est donc probablement sous-estimée.

D'après nos estimations, la production totale de chitine par les Crustacés dans les écosystèmes marins à l'échelle de la planète serait de 2 milliards 300 millions de tonnes par $a^{4}$. Cette production serait imputable, pour près de $90 \%$, aux organismes pélagiques, Crustacés du zooplancton et krill. La contribution des autres organismes animaux producteurs de chitine apparaît comme négligeable par rapport à celle des Crustacés.

Il est intéressant de comparer cette estimation chiffrée à d'autres estimations basées sur d'autres considérations, comme sur le rendement des efforts de

(4) Et non de « 2.3 millions metric tons per year » comme indiqué erronément dans une publication antérieure (Jeuniaux et VossFoucart, 1991; Chitin biomass and production in the marine environment ; Biochemical Systematics and Ecology, 19, fasc. 5, p. 347 et 254).

Vol. 6, $\mathrm{n}^{\circ} 4-1993$ pêche industrielle, par exemple. Pour Ross et Quétin (1988), la production annuelle de chitine par le krill serait de 10 à $70 \mathrm{~g}$ par $\mathrm{m}^{2}$ de surface océanique, une valeur hypothétique bien plus élevée que celle que nous avons retenue (5.3), mais considérée par d'autres auteurs comme nettement surévaluée. Quant à Allan et al. (1978), ils estiment à $39 \cdot 10^{3}$ tonnes par an la production de chitine par les grandes espèces de Crustacés Décapodes, ce qui est largement inférieur à notre évaluation (2 millions de tonnes); il faut noter cependant que les estimations de ces auteurs sont basées sur les statistiques des pêcheries de Crabes et de Langoustes, qui sont loin d'exploiter l'ensemble des milieux susceptibles d'héberger ces espèces.

\section{CONCLUSIION}

L'estimation de 2.3 milliards de tonnes de chitine produites par an par les Crustacés marins, toute approximative qu'elle soit assurément, donne une idée de l'importance de ce polysaccharide dans les cycles biogéochimiques de l'azote et du carbone. Ces quantités énormes de chitine produites chaque année dans les mers et les océans constitueraient à la longue une immobilisation importante du carbone et de l'azote biogènes, sans l'existence de mécanismes de biodégradation efficaces. Ceux-ci impliquent la mise en oeuvre d'enzymes chitinolytiques qui agissent à plusieurs niveaux différents. (1) D'une part, dans la chaîne trophique des consommateurs, grâce à la sécrétion de chitinases et de chitobiases par les organes du système digestif de la plupart des animaux qui consomment des proies à téguments chitineux (pour une revue de cette question, voir Jeuniaux, 1963, 1971, 1978). (2) D'autre part, chez les Crustacés, grâce à la libération d'enzymes chitinolytiques par l'épiderme qui permettent l'hydrolyse et la récupération d'une partie de la chitine cuticulaire pendant les mues, et qui interviennent aussi après la mort des organismes (Poulicek et Jeuniaux, 1991). Enfin, pendant la dégradation microbienne qui accompagne l'incorporation dans les sédiments des cadavres, déchets et exuvies (Gooday, 1990, 1991 ; Poulicek et Jeuniaux, 1991).

\section{RÉFÉRENCES}

Allan G. G., J. R. Fox, N. Kong, 1978. Marine polymers : part 8: A critical evaluation of the potential sources of chitin and chitosan. In : R. A. A. Muzzarelli, E. R. Pariser, ed., Proc. First Internat. Conf. Chitin/Chitosan, MT Sea Grant Program, 78-7, Massachussetts, 68-78.

Anderson C. G., N. de Pablo, C. R. Romo, 1978. Antarctic krill (Euphausia superba) as a source of Chitin and Chitosan. Proc. First Internat. Conf. Chitin/Chitosan, R. A. A. Muzzarelli, E. R. Pariser, ed., MIT Sea Grant Program, 78-7, Massachussetts, 54-63. 
Berger L. R., D. M. Reynolds, 1958. The chitinase system of a strain of Streptomyces griseus. Biochim. Biophys. Acta, 29, 522-534.

Berry P. F., M. J. Smale, 1980. An estimate of production and consumption rates in the spiny lobster Panulirus homarus on a shallow littoral reef off the Natal Coast, South Africa, Mar. Ecol. Progr. Ser., 2, 337- 343.

Brzeski M. M., 1982. Concept of Chitin/Chitosan isolation from antarctic krill (Euphausia superba Dana) shells on a technical scale. In : Chitin and Chitosan, S. Hirano, S. Tokura, eds., Sapporo, Japan, 15-21.

Bussers J.-C., Ch. Jeuniaux, 1974. Recherche de la chitine dans les productions métaplasmatiques de quelques Ciliés. Protistologica, 10, 43-46.

Bussers J.-C., M. Poulicek, J. Diepvints, 1983. Description d'une suçeuse à air comprimé économique et utilisable par un seul plongeur. Cah. Biol. Mar., 24, 215-217.

Campbell F. L., 1928. The detection and estimation of insect chitin and the irrelation of chitinization to hardness and pigmentation of the cuticula of the American cockroach Periplaneta americana. Ann. Entom. Soc. Am., 22, 401426.

Darmon S. E., K. M. Rudall, 1950. Infra-red and X-ray studies of chitin. Discuss. Faradey Soc., 9, 251.

Dauby P., 1985. Plankton productivity in the gulf of Calvi (Corsica). Progr. Belgian Oceanogr. Res., Brussels, 442450.

Dauby P., Ch. Jeuniaux, 1986. Origine exogène de la chitine décelée chez les Spongiaires. Cah. Biol. Mar., 28, 121-129.

Everson I., 1977. The living Resources of the Southern Ocean, Ocean Fisheries Survey Programme, United Nations Development Program, F.A.O. Roma.

Gervasi E., Ch. Jeuniaux; P. Dauby, 1988. Production de chitine par les crustacés du zooplancton de la baie de Calvi (Corse), In: Aspects récents de la Biologie des Crustacés, IFREMER ed., Brest, France, 33-38.

Giles C. H., A. S. A. Hassan, M. Laidlaw, R. V. R. Subramanian, 1958. J. Soc. Dyers Colourists, 74, 647.

Goffinet G., Ch. Jeuniaux, 1979. Distribution et importance quantitative de la chitine dans les coquilles de Mollusques. Cah. Biol. Mar., 20, 341-349.

Goldstein I. J., S. Hammarström, G. Sunblad, 1975. Precipitation and carbohydrate-binding specificity studies on wheat germ agglutinin. Biochim. Biophys. Acta, 405, 53-61.

Gooday G. W., 1990. The ecology of chitin degradation. In : Advances in microbial ecology, vol. 11, K. C. Marshall ed., Plenum Press, 387-440.

Gooday G. W., J. I. Prosser, K. Hillman, M. G. Cross, 1991. Mineralization of Chitin in an Estuarine Sediment : The importance of the Chitosan Pathway. Biochem. System. Ecol., 19, 5, 395-400.

Hackman R. H., 1960. Studies on Chitin. IV. The occurrence of complexes in which chitin and protein are covalently linked. Austr. J. Biol. Sci., 13, 568-577.

Hackman R. H., 1982. The Estimation of chitin in Arthropod cuticules. In : Chitin and Chitoșan, Proc. $\Pi^{\text {nd }}$ Int. Conf. on Chitin and Chitosan, Sapporo (Japan), 5-9.

Hedley R. H., 1962. The significance of an "inner chitinous lining" in Saccamminid organisation with special reference to a new species of Saccamina (Foram.) from New Zealand. New Zealand I. Sci., 5, 375-389.
Hirano S., Y. Koishibara, S. Kitaura, T. Taneko, H. Tsuchida, K. Murae, T. Yamamoto, 1991. Chitin biodegradation in Sand Dunes. Biochem. System. Ecol., 19, 379-384.

Horisberger M., J. Rosset, 1977. Colloïdal gold a useful marker for transmission and scanning electron microscopy. J. Histochem. Cytochem., 25, 295-305.

Jeuniaux Ch., 1958. Recherches sur les chitinases. I : dosage néphélométrique et production de chitinase par des Streptomycètes. Arch. Internat. Physiol. Biochim., 66, 408-427.

Jeuniaux Ch., 1959. Recherches sur les chitinases. II : purification de la chitinase d'un Streptomycète et séparation électrophorétique de principes chitinolytiques distincts. Arch. Internat. Physiol Biochim., 57, 597- 617.

Jeuniaux Ch., 1961. Chitinase : an addition to the list of hydrolases in the digestive tract of Vertebrates. Nature (London), 192, 135-136.

Jeuniaux Ch., 1963. Chitine et chitinolyse, un chapitre de la biologie moléculaire. Paris, Masson, $181 \mathrm{p}$

Jeuniaux Ch., 1965. Chitine et phylogénie : application d'une méthode enzymatique de dosage de la chitine, Bull. Soc. Chim. Biol., 47, 2267-2278.

Jeuniaux Ch., 1966. Chitinases. In : Methods in Enzymology, VIII, Complex Carbohydrates ; Neufeld, Ginsburg ed., Academic Press, 8, 644-650.

Jeuniaux Ch., 1971. On some biochemical aspects of regressive evolution in animals. In: Biochemical Evolution and the Origin of Life, E. Schoffeniels, ed., Elsevier North-Holland, 304-313.

Jeuniaux Ch., 1982a. La chitine dans le règne animal. Bull. Soc. Zool. France, 107, 363-386.

Jeuniaux Ch., 1982b. Composition chimique comparée des formations squelettiques chez les Lophophoriens et les Endoproctes. Bull. Soc. Zool. France, 107, 233-249.

Jeuniaux Ch., G. Dandrifosse, J. C. Micha, 1982. Caractères et évolution des enzymes chitinolytiques chez les Vertébrés inférieurs. Biochem. Syst. Ecol., 10, 365-372.

Jeuniaux Ch., M. F. Voss-Foucart, J. C. Bussers, 1982. Preliminary results on chitin biomass in some benthic marine biocenoses. In : Chitin and Chitosan, S. Hirano, S. Tokura ed., Sapporo, Japan, 12-14, 200-204.

Jeuniaux Ch., M. F. Voss-Foucart, J. C. Bussers, 1991. Chitin Biomass and Production in the Marine Environment. Biochem. System. Ecol., 19, 5, 347-356.

Jeuniaux Ch., J. C. Bussers, M. F. Voss-Foucart, M. Poulicek, 1986. Chitin production by animals and natural communities in marine environment. In : Chitin in Nature and Technology, Muzzarelli, Jeuniaux, Gooday, ed., Plenum Press, N. Y., 515- 522.

Jeuniaux Ch., M. F. Voss-Foucart, E. Gervasi, J. C. Bussers, M. Poulicek, 1988. Biomasse et production de chitine par des biocénoses benthiques et planctoniques de la baie de Calvi. Bull. Soc. R. Sci. Liège, 57, 287-299.

Jeuniaux Ch., M. F. Voss-Foucart, M. Poulicek, J. C. Bussers, 1989. Sources of chitin, estimated from new data on chitin biomass and production. In : Skjak-Braek et al., ed., Chitin and chitosan, Elsevier (London), 57, 3-11.

Lindley J. A., 1982. Continuous Plankton records : geographical variations in numerical Abundance, Biomass and Production of Euphausiids in the North Atlantic Ocean and the North Sea. Mar. Biol., 71, 7-10.

Micha J. C., G. Dandrifosse, Ch. Jeuniaux, 1973. Distribution et localisation tissulaire des chitinases chez 
les Vertébrés inférieurs. Arch. intern. Physiol. Biochim., 81, 439-451.

Miller R. F., 1991. Chitin Paleoecology, Biochem. System. Ecol., 19, 401-411.

Milliman J. D., 1974. Marine Carbonates, Springer Verlag, $375 \mathrm{p}$.

Miyamoto S., H. Yamamoto H., Seki, 1991. Chitin Dynamics in Freshwater Environment, Biochem. Syst. Ecol., 19, 371-377.

Ohtakara A., 1971. The chitinase system of a strain of Streptomyces griseus. Hiroshima Women's Univ., Natur. Sci., 6, 1-12.

Okutani K., 1975. Microrganisms related to mineralization of chitin in aquatic environments. In : Nitrogen fixation and Nitrogen Cycle, H. Takahashi, ed. Univ. Tokyo Press, 147-154.

Pearson F. G., R. H. Marchessault, C. Y., Lyang, 1960. Infrared spectra of crystalline polysaccharides. 5 : Chitin. J. Polym. Sc., 43, 101-116.

Peters W., 1966. Chitin in Tunicata. Experientia, 22, 820-821.

Peters W., 1967. Zur Frage des Vorkommens und der Definition peritrophischer membranen. Verh. Deutsch. Zool. Gesell., Göttingen, 30 suppl., 142-152.

Poulicek M., 1983. Patterns of mollusk shell biodegradation in bathyal and abyssal sediments. Molluscan Study, 12A, 136-141.

Poulicek M., M. F. Jaspar-Versali, 1984. Biodégradation de la trame organique des coquilles de Mollusques en milieu marin : action des microorganismes endobiontes. Bull. Soc. R. Sci. Liège, 53, 114-126.

Poulicek M., Ch. Jeuniaux, 1989. Chitin Biomass in marine sediments. In : Chitin and Chitosan, G. Skjak-Brack, T. Anthonsen, P. Sandford, ed., Elsevier Applied Science, 151-155.

Poulicek M., Ch. Jeuniaux, 1991. Chitin Biodegradation in Marine Environments : an experimental approach. Biochem. System. Ecol., 19, 385-394.

Poulicek M., G. Goffinet, M. F. Voss-Foucart, J. C. Bussers, C. Toussaint, 1986. Chitin degradation in natural environment (mollusk shells and crab carapaces). In : Chitin in Nature and Technology, R. M. Muzzarelli, Ch. Jeuniaux, G. Gooday, ed. Plenum Press (N. Y.), 547-550.
Reissig J. L., J. L. Strominger, L. F. Leloir, 1955. A modified colorimetric method for the estimation of N-Acetylamino sugars. J. Biol. Chem., 217, 959-966.

Ritz D. A., G. W. Hosie, 1982. Production of the Euphausiid Nyctiphanes australis in Storn Bay, SouthEastern Tasmania. Mar. Biol., 68, 103- 108.

Roberts R. L., E. Cabib, 1982. Serratia marcescens Chitinase: one-step purification and use for the determination of chitin. Analyt. Biochem., 127, 402-412.

Ross R. M., L.B. Quétin, 1988. Euphausia superba : a critical review of estimates of annual production. Comp. Biochem. Physiol., 90B, 499-505.

Rudall K. M., 1963. The chitin/protein complexes of insect cuticles. Adv. Insect. Physiol., 1, 257-313.

Sachs J.B., 1954. The chemical nature of the cyst membranes of Pelomyxa illinoisensis. J. Protoz., 1, 8-9.

Seki H., 1965. Microbiological studies on the decomposition of chitin in marine environment. IX, X:J. Oceanogr. Soc. Japan, 21, 17-33.

Seki H., 1966. Seasonal fluctuation of heterotrophic bacteria in the sea of Aburatsubo Inlet. J. Oceanogr. Soc. Japan, 22, $15-16$

Seki H., N. Taga, 1963. Microbiological studies on the decomposition of chitin in marine environment, I, II, III : J. Oceanogr. Soc. Japan, 19, 101- 151.

Smucker R. A., 1991. Chitin primary Production. Biochem. System. Ecol., 19, 357-369.

Tchernia P., 1978. Oceanographie régionale. Description physique des océans et des mers. Ecole Normale Supérieure Techniques Avancées, Paris.

Vavra J., 1967. Hydrolyse enzymatique des spores de Microsporidies. J. Protoz., 14, suppl. 49.

Voss-Foucart M. F., J. C. Bussers, R. Poizat, Ch. Jeuniaux, 1991. Etude quantitative préliminaire de la composition chimique des communautés pionnières colonisant divers types de plastiques en Méditerranée. Rapp. Comm. Int. Mer Médit., 32, 27.

Winberge G. G., 1971. Methods for estimation of production of aquatic animals. Academic Press, London, 175 p.

Yanase M., 1975. Chemical composition of the Exoskeleton of Antarctic Krill. Bull. Tokai Reg. Fish Res. Lab., 83, 1 - 6.

Zobell C. E., S. C. Rittenberg, 1938. The occurrence and characteristics of chitinoclastic bacteria in the sea. J. Bact., $35,275-287$. 